Ayso H. de Vries

Marjolein H. Liedenbaum

Shandra Bipat

Roel Truyen

Iwo W. O. Serlie

Rutger H. Cohen

Saskia G. C. van Elderen

Anneke Heutinck

Oskar Kesselring

Wouter de Monyé

Lambertus te Strake

Tjeerd Wiersma

Jaap Stoker

Received: 27 August 2008

Revised: 1 January 2009

Accepted: 26 January 2009

Published online: 20 March 2009

(C) The Author(s) 2009. This article is published with open access at Springerlink.com

A. H. de Vries $(\bowtie)$.

M. H. Liedenbaum - S. Bipat .

A. Heutinck - J. Stoker

Department of Radiology Academic

Medical Centre,

University of Amsterdam,

Meibergdreef 9,

1105 AZ Amsterdam, The Netherlands

e-mail: Ayso.devries@amc.uva.nl

e-mail: aysodevries@hotmail.com

Tel.: +31-020-56663028

Fax: +31-020-56669119

R. Truyen · I. W. O. Serlie

Department of Healthcare Informatics,

Philips Medical Systems 3,

Veenpluis 6 ,

5684 PC Best, The Netherlands

R. H. Cohen

Department of Radiology,

Ziekenhuis Amstelland,

Laan van de Helende Meesters 8,

1186 AM

Amstelveen, The Netherlands

S. G. C. van Elderen

Department of Radiology,

Leids Universitair Medisch Centrum,

Albinusdreef 2

2333 ZA Leiden, The Netherlands

\section{Primary uncleansed 2D versus primary electronically cleansed 3D in limited bowel preparation CT-colonography. Is there a difference for novices and experienced readers?}

\author{
O. Kesselring $\cdot$ T. Wiersma \\ Department of Radiology, \\ Ziekenhuis Rijnstate, \\ Wagnerlaan 55, \\ 6815 AD Arnhem, The Netherlands \\ W. de Monyé \\ Department of Radiology, \\ Kennemer Gasthuis, \\ Boerhaavelaan 22, \\ 2035 RC Haarlem, The Netherlands \\ L. te Strake \\ Department of Radiology, \\ Middlemore Hospital, \\ Private Bag 93311, \\ Otahuhu, Auckland, 6, New Zealand
}

\begin{abstract}
The purpose of this study was to compare a primary uncleansed 2D and a primary electronically cleansed 3D reading strategy in CTC in limited prepped patients. Seventytwo patients received a low-fibre diet with oral iodine before CTcolonography. Six novices and two experienced observers reviewed both cleansed and uncleansed examinations in randomized order. Mean per-polyp sensitivity was compared between the methods by using generalized estimating equations. Mean per-patient sensitivity, and
\end{abstract}

specificity were compared using the McNemar test. Results were stratified for experience (experienced observers versus novice observers). Mean per-polyp sensitivity for polyps $6 \mathrm{~mm}$ or larger was significantly higher for novices using cleansed 3D (65\%; 95\% CI 57-73\%) compared with uncleansed 2D (51\%; 95\%CI 44$59 \%$ ). For experienced observers there was no significant difference. Mean per-patient sensitivity for polyps $6 \mathrm{~mm}$ or larger was significantly higher for novices as well: respectively $75 \%$ $(95 \%$ CI $70-80 \%)$ versus $64 \%$ (95\%CI 59-70\%). For experienced observers there was no statistically significant difference. Specificity for both novices and experienced observers was not significantly different. For novices primary electronically cleansed $3 \mathrm{D}$ is better for polyp detection than primary uncleansed 2D.

Keywords CT-colonography · Sensitivity and specificity - Colonic polyps - Electronic cleansing · Colorectal neoplasms

\section{Introduction}

CT-colonography (CTC) has consistently been shown to have a high accuracy for the detection of colorectal neoplasia, and has recently been included in the official guidelines for colorectal cancer screening [1]. An im- portant disadvantage of the technique is that many patients find the bowel preparation burdensome [2]. Therefore efforts have been made to prepare patients for CTC with a less extensive bowel preparation [3-7]. Minimizing bowel preparation may increase patient compliance [8-10], but will result in larger amounts of residual faeces in the colon. 
A prerequisite is that faecal material is labelled with oral contrast (i.e. faecal tagging) in order to differentiate faecal material from colonic structures.

To our knowledge, all limited prepared CTC studies have been performed using primary two-dimensional (2D) display methods [3-5]. The rationale for this approach is that submerged segments can be better assessed in 2D.

Previous studies in patients with extensive bowel preparation have indicated that primary three-dimensional (3D) reading may result in less false negative findings compared with primary $2 \mathrm{D}$ reading $[11,12]$. If a similar empty endoluminal view could be achieved by electronic removal of tagged material ("electronic cleansing") in patients who have undergone limited bowel preparation, primary 3D could be a method of choice.

However, specific artefacts of electronic cleansing were described that potentially reduced the accuracy of CTC [13]. This may be the reason for the paucity of papers on the use of electronic cleansing. A specifically noticeable problem is posed by the distracting 'ridges' or 'pseudopolyps' emanating from locations where air, soft tissue and tagged material meet.

We hypothesized that especially for inexperienced observers a primary electronically cleansed 3D (PEC3D) method may have advantages for evaluation of the colon: polyps are visible for longer than in a $2 \mathrm{D}$ method and PEC3D provides a more intuitive reproduction of reality. In this study we used a cleansing algorithm that was devised to improve 3D image quality at the junctions of air, soft tissue and tagged material.

Therefore the purpose of this study was to assess whether there was any difference in accuracy between two different reading strategies for the detection of colorectal polyps in a patient population that had undergone a reduced bowel preparation. The results of primary uncleansed $2 \mathrm{D}$ (PU2D) and primary electronically cleansed 3D (PEC3D) were stratified for reader experience.

\section{Materials and methods}

Study population

The institutional review board of our hospital approved the study. All patients gave written informed consent.

The CT datasets used in this study were a consecutive series of FOBT (faecal occult blood test) positive patients that were included in the framework of a previous comparative study of two different faecal occult blood tests [14].

\section{Bowel preparation}

Bowel preparation started 2 days before CTC and consisted of seven $50-\mathrm{ml}$ aliquots of meglumine ioxithalamate
(Telebrix Gastro 300 mg I/ml; Guerbet, Cedex, France) administered orally (undiluted) with each meal (breakfast, lunch and dinner). The use of oral contrast was combined with a low-fibre diet. The evening and morning before the CTC examination no solid foods were allowed. Explicit instructions about fluid intake were not given. No laxatives were used in order to minimize patient discomfort.

\section{Scan parameters}

CTC was executed according to state-of-the-art techniques. Patients were examined in prone and supine position after the intravenous administration of bowel relaxants (Buscopan; Boehringer Ingelheim, Germany or, if contraindicated, Glucagon; Novo-Nordisk, Bagsvaerd, Denmark). $\mathrm{CO}_{2}$ was automatically insufflated $\left(\mathrm{PROTOCO}_{2} \mathrm{~L}, \mathrm{EZ}-\mathrm{E}-\mathrm{M}\right)$. Patients were not given intravenous contrast medium. Examinations were performed on a 64-slice multidetector CT system (Brilliance, Philips Medical Systems, Best, the Netherlands) with a reference $\mathrm{mA} \mathrm{s}$ value of $40 \mathrm{~mA} \mathrm{~s}(z$-axis tube modulation and automatic current selection was used). Collimation was $64 \times 0.625 \mathrm{~mm}$, pitch 1.2 , slice thickness $0.9 \mathrm{~mm}$, rotation time $0.4 \mathrm{~s}$ and tube voltage $120 \mathrm{kV}$.

Reading methods

The examinations were read in random order i.e. the PU2D and PEC3D datasets were interspersed. The observers were blinded to the results of the reference standard. To avoid recall bias, we aimed to maximize the interval between the PU2D and PEC3D reading of the same patient. This period varied per observer (mean 33 days, range 7-66) (Table 1). All detected lesions were recorded in a digital database. The method of detection (PU2D or PEC3D), colon segment and size of the lesion (as measured in the primary review method) of all findings were documented.

\section{Primary uncleansed 2D (PU2D)}

The PU2D interface is illustrated in Fig. 1 (ViewForum 6.1, Philips Medical Systems), using axial CT images ( $W 1,250$, $L$ 50). Observers were free to adjust the window setting when appropriate. To further elucidate suspected findings on 2D, an uncleansed 3D display and a 3D colon overview could be viewed for problem solving. The dual-screen interface simultaneously displayed both supine and prone scan positions.

\section{Primary electronically cleansed 3D (PEC3D)}

The PEC3D interface is illustrated in Fig. 2. The unfolded cube review method (ViewForum 6.1, Philips Medical 
Table 1 Individual observer performance in PU2D and PEC3D

\begin{tabular}{|c|c|c|c|c|c|c|}
\hline \multicolumn{2}{|c|}{ Observer } & \multirow[t]{2}{*}{$\begin{array}{l}\text { Number of primary 2D CTCs } \\
\text { verified by colonoscopy }\end{array}$} & \multirow[t]{2}{*}{$\begin{array}{l}\text { Number of primary } 3 \mathrm{D} \text { CTCs } \\
\text { verified by colonoscopy }\end{array}$} & \multicolumn{2}{|c|}{$\begin{array}{l}\text { Review } \\
\text { times (s) }\end{array}$} & \multirow[t]{2}{*}{$\begin{array}{l}\text { Mean interval (days) between both } \\
\text { review methods (SD) }\end{array}$} \\
\hline \multicolumn{2}{|c|}{ Number Experience } & & & $2 \mathrm{D}$ & $3 \mathrm{D}$ & \\
\hline 2 & \multicolumn{2}{|c|}{ Experienced 500} & 100 & 574 & 593 & $32(15)$ \\
\hline 6 & \multicolumn{2}{|c|}{ Experienced 300} & 50 & 432 & 579 & $66(38)$ \\
\hline \multicolumn{4}{|c|}{ Mean experienced observer } & \multicolumn{3}{|c|}{$503 * 586 *$} \\
\hline 1 & Novice & 40 & 10 & 765 & 870 & $11(4)$ \\
\hline 3 & Novice & 40 & 10 & 756 & 964 & $36(19)$ \\
\hline 4 & Novice & 40 & 10 & 404 & 376 & $7(3)$ \\
\hline 5 & Novice & 40 & 10 & 259 & 419 & $60(38)$ \\
\hline 7 & Novice & 40 & 10 & 634 & 795 & $30(23)$ \\
\hline 8 & Novice & 40 & 10 & 403 & 447 & $20(9)$ \\
\hline \multicolumn{4}{|c|}{ Mean novice observers } & \multicolumn{3}{|c|}{$537 * 645 *$} \\
\hline
\end{tabular}

$S D$ standard deviation

*Denotes statistically significant difference between the review methods of both groups

Systems) was developed to maximize the area of visible colon surface and was previously validated [15]. The unfolded cube display method was simultaneously displayed with corresponding original-uncleansed-2D multiplanar reformatted images and a 3D colon overview for problem solving. The dual-screen interface simultaneously displayed both supine and prone examinations.

At the time of study the cleansing algorithm (ViewForum, Philips Medical Systems, Best, the Netherlands) [16] was not yet commercially available. In short, the algorithm assumes that the measured density in a voxel arises due to a combination of three materials: soft tissue, air and tagged material. Initially, the percentage of materials in each voxel is determined. Subsequently, the partial volume of tagged material is replaced by air and the new density is calculated. Finally, a 3D method visualizes the colon from an endoluminal perspective as if there were no faecal remains.

During the study the algorithm was not yet integrated into the system and was therefore processed on a separate computer (Precision 690, Dell, Round Rock, USA). Afterwards, the cleansed data were reloaded on the workstation.

\section{Observers}

Eight physicians participated in this study: two experienced observers (two research physicians working full time on

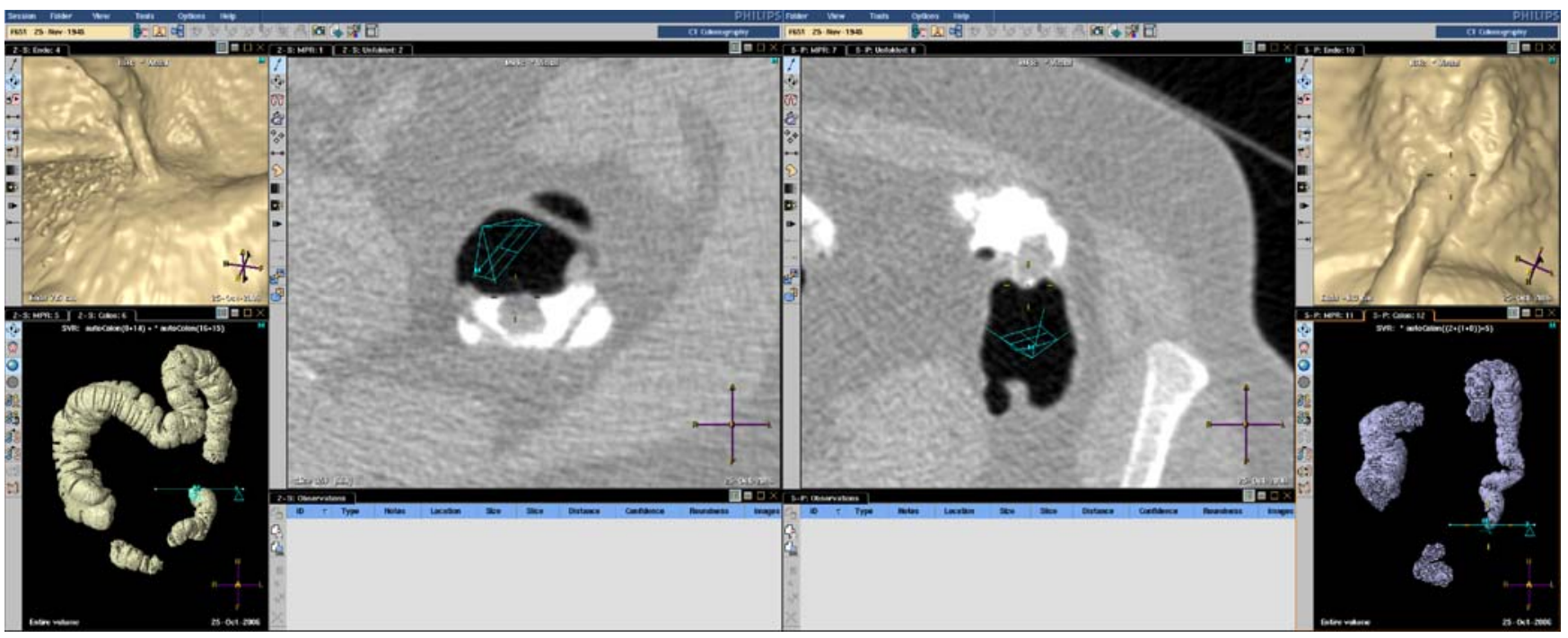

Fig. 1 Interface of the PU2D method. Uncleansed 3D images were used for problem solving. In the corresponding scan positions a large stalked polyp can be seen in the descending colon 


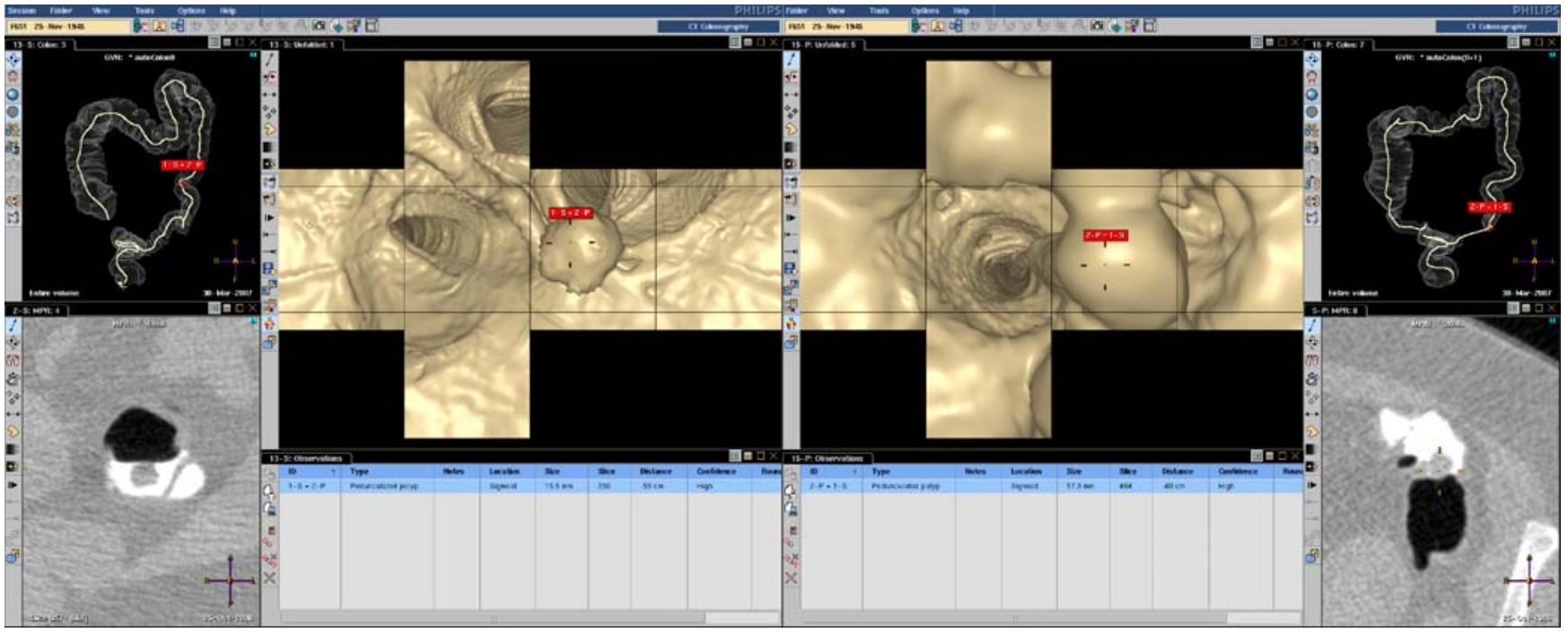

Fig. 2 Interface of the PEC3D method. Uncleansed 2D images were used for problem solving. In the corresponding scan positions the same large stalked polyp can be seen as in Fig. 1. This submerged polyp has become visible in 3D after electronic cleansing

CTC) and six novice observers (five radiologists, one recently qualified physician).

The two experienced observers had seen over $350 \mathrm{CTC}$ examinations verified by colonoscopy (among these the 75 patients included in this study). All novice observers, without any notable prior experience with CTC, had undergone the same learning curve. The learning curve consisted of 50 selected CTC examinations from a publicly available database [17]. Forty examinations were read using a primary $2 \mathrm{D}$ method and 10 using a primary $3 \mathrm{D}$ method. The ratio for this distribution was the assumption that detecting lesions in $2 \mathrm{D}$ is more difficult than in $3 \mathrm{D}$, although the assessment of a suspected lesion is similar in both review methods. Personal feedback on false negative and false positive findings for polyps $6 \mathrm{~mm}$ or larger was provided by a radiology research fellow (more than 500 CTC examinations verified by colonoscopy).

The observers were aware of the fact that the actual study was done on FOBT positive patients.

\section{Reference standard}

The reference standard was based on the findings of the initial CTC reading, which was double read by two experienced observers (more than 200 CTC) and verified by colonoscopy with segmental unblinding. Colonoscopy was performed within 2 weeks after CTC.

A polyp seen during CTC was considered true positive if (1) its appearance resembled the corresponding adenomatous or nonadenomatous polyp at colonoscopy, (2) its segment or adjacent segment corresponded with the segment of the reference standard and (3) the polyp size as estimated by the endoscopist corresponded with the CTC size, considering a margin of error of $50 \%$. Since the colonoscopy measurement is subject to inaccuracy $[18,19]$ this criterion could be overruled by the first two criteria. All other annotations were considered false positives.

The relation of polyps to faecal material

To illustrate the influence of faecal material on the visibility of polyps, a research fellow determined whether each polyp was completely covered by faecal material (i.e. completely submerged in both positions), partially covered by faecal material (i.e. not covered in both scan positions but at least partially covered in at least one position) or not covered by faecal material at all.

Power analysis

A power calculation was performed based on an assumed $15 \%$ difference between the methods in sensitivity for polyps $6 \mathrm{~mm}$ or larger (i.e. $70 \%$ versus $85 \%$ ). The number of visible polyps required to detect a statistically significant difference by using the McNemar test was $75(p=0.05)$. As we expected some of the patients to be excluded due to insufficient diagnostic quality, we included a total of 75 examinations of FOBT positive patients that had 84 visible polyps.

\section{Performance}

Per polyp To investigate differences between PU2D and PEC3D, we calculated the mean per-polyp sensitivity for both experienced and novice readers. Statistical differences between the review methods were assessed by using 
generalized estimating equations (GEE) (SPSS, 15.0, Statistics, Chicago, USA) to revise for data clustering and dependency. In this GEE method, regression analyses were performed to compare the mean sensitivity values of the two methods [20]. Since the per-polyp specificity cannot be calculated because it is a nonexisting entity, we confined the per-polyp results to the number of false positive findings.

Per patient Other main outcome per-patient parameters were per-patient sensitivity and specificity. Statistical differences in mean per-patient sensitivity and mean specificity measures were assessed with the McNemar test.

Both mean per-polyp and per-patient outcome measures were analysed according to cutoff values of 6 and $10 \mathrm{~mm} . P$ values $<0.05$ were considered statistically significant.

\section{Review time}

The review time, defined as the time measured with a stopwatch to review a complete examination, was compared for both methods. The review time did not include the time required for processing the images. These procedures are highly dependent on calculation power, are performed semiautomatically and require no reviewer interaction.

Differences in mean review time of experienced and novice observers were assessed with a paired Student's $t$ test. $P$ values $<0.05$ were considered statistically significant.

\section{Image quality}

The image quality of the examinations was rated (after reading) on a four-point Likert scale: diagnostic without artefacts; diagnostic with a small number of artefacts, polyps $6 \mathrm{~mm}$ or larger cannot be missed; diagnostic with many artefacts, polyps 6-9 $\mathrm{mm}$ can be missed; not diagnostic, polyps $10 \mathrm{~mm}$ or larger can be missed.

Firstly, if at least four observers rated the examination as "not diagnostic" the patient was excluded.

Secondly, we determined the percentage of PU2D and PEC3D examinations in the various rating categories. The percentage represented the mean rating of all eight observers.

Thirdly, we assessed per-observer differences in quality between PU2D and PEC3D using ordinal regression analysis with PU2D as reference standard. A lower relative diagnostic odds ratio (RDOR) implies inferior image quality of the PEC3D compared with the PU2D. Confidence intervals not reaching 1 indicate significant inferiority.

Fourthly, each observer determined the presence of artefacts per examination. The impact of each artefact on the "readability" of the examination was assessed on a four-point Likert scale: not disturbing; disturbing, but cannot hinder detection of polyps $6 \mathrm{~mm}$ or larger; disturbing, can cover polyps 6-9 $\mathrm{mm}$; and disturbing, can cover polyps $10 \mathrm{~mm}$ or larger. We report the number of patients with artefacts observed by at least four observers and the number of patients with artefacts classified by at least four observers as "disturbing, can cover polyps". These were analysed according to cutoff values of 6 and $10 \mathrm{~mm}$.

\section{Results}

All 75 patients were scanned between October 2006 and January 2007 and underwent colonoscopy within 9.4 days (SD 6.6 days). We excluded three of the 75 patients because more than four observers rated the diagnostic quality of three PEC3D examinations "not diagnostic". In 2D these examinations were rated by one to three observers as "not diagnostic". The remaining 72 patients consisted of 38 men and 34 women (mean age 59.5 years, SD 6.4 years, range 50-73). Bleeding during polypectomy was reported for three patients, none of whom required reintervention. No adverse events were reported to occur in any of the CT examinations.

A total of 90 polyps $6 \mathrm{~mm}$ or larger were detected: 17 polyps were $20 \mathrm{~mm}$ or larger (median size $25 \mathrm{~mm}$, range 20-40 mm), 36 polyps were 10-19 mm (median size $11 \mathrm{~mm}$, range $10-17 \mathrm{~mm}$ ) and 37 polyps were $6-9 \mathrm{~mm}$ (median size $7 \mathrm{~mm}$, range 6-9 $\mathrm{mm}$ ). The histology revealed 82 adenomatous polyps, 4 nonadenomatous polyps and 4 colorectal carcinomas.

Polyps $6 \mathrm{~mm}$ or larger were found in 50 out of 72 patients $(69 \%)$ and polyps $10 \mathrm{~mm}$ or larger were found in 34 out of 72 patients (47\%). As shown by Table 2 a substantial part of most polyps was covered by faecal material.

\section{Performance}

Per polyp Mean per-polyp sensitivity for novices and experienced observers for both review methods are listed in Table 3. Novice observers had a significantly higher mean sensitivity when using PEC3D for polyps $6 \mathrm{~mm}$ or larger $(+14 \%, p<0.001), 6-9 \mathrm{~mm}(+19 \%, p<0.001)$ and $10 \mathrm{~mm}$ or larger $(+9 \%, p<0.001)$. Within the group of novice observers there was a considerable difference in sensitivity for both review methods (Tables 3 and 4), despite the fact that all observers had undergone a similar training protocol.

For experienced observers there was no significant difference in mean sensitivity between both methods for polyps $6 \mathrm{~mm}$ or larger $(p=0.755), 6-9 \mathrm{~mm}(p=0.170)$ and $10 \mathrm{~mm}$ or larger $(p=0.207)$. 
Table 2 Relation of polyps to faecal material

\begin{tabular}{|c|c|c|c|c|}
\hline & \multicolumn{2}{|c|}{ 6-9 mm } & \multicolumn{2}{|c|}{$\geq 10 \mathrm{~mm}$} \\
\hline & $n$ & $\%$ & $n$ & $\%$ \\
\hline Polyps completely covered by faecal material in both scan positions & 1 & 3 & 2 & 4 \\
\hline Polyps partially covered by faecal material in one or both scan positions or completely covered in one position & 13 & 35 & 28 & 53 \\
\hline Polyps not covered by faecal material at all in both scan positions & 18 & 49 & 22 & 42 \\
\hline In retrospect not visible in both scan positions & 5 & 14 & 1 & 2 \\
\hline Total number of polyps & 37 & 100 & 53 & 100 \\
\hline
\end{tabular}

Table shows the number $(n)$ and percentage of visible polyps either completely covered by faecal material, partially covered by faecal material or not covered by faecal material at all in two scan positions. Polyps that are not visible at all are reported as well

Per patient The per-patient performance characteristics for PU2D and PEC3D are shown in Table 4. Novice observers had a significantly higher mean sensitivity when using PEC3D for polyps $6 \mathrm{~mm}$ or larger $(+11 \%, p<0.001)$ and $10 \mathrm{~mm}$ or larger $(+6 \%, p=0.033)$ compared with PU2D. For experienced observers there was no significant difference in mean sensitivity between both methods for polyps $6 \mathrm{~mm}$ or larger $(p=0.549)$ and $10 \mathrm{~mm}$ or larger $(p=0.125)$.

Specificity for novice observers when using PEC3D was not significantly lower for polyps $6 \mathrm{~mm}$ or larger $(p=0.057)$ and $10 \mathrm{~mm}$ or larger $(p=0.36)$ compared with PU2D. For experienced observers there was no significant difference for polyps $6 \mathrm{~mm}$ or larger $(p=0.5)$ and $10 \mathrm{~mm}$ or larger $(p=1.0)$ as well. Thus, specificity did not significantly differ between both methods in any size category for both experienced and novice observers.

\section{Review time}

For novice observers mean review time for PU2D was $8.9 \mathrm{~min}$ (range 4.3-12.8 min) compared with $10.8 \mathrm{~min}$ (range $6.3-16.1 \mathrm{~min})$ for PEC3D $(p<0.001)$. For experienced observers the review times were respectively $8.4 \mathrm{~min}$ (range 7.2-9.6 $\mathrm{min}$ ) and $9.8 \mathrm{~min}$ (range 9.6-9.9 $\mathrm{min})(p<0.001)$.

For most observers PU2D was faster. One observer evaluated the examinations faster in primary cleansed $3 \mathrm{D}$ method (Table 1).

\section{Diagnostic quality}

The mean rating of the diagnostic quality is displayed in Table 5. Figure 3 shows that all observers rated the image quality of PEC3D significantly lower than PU2D, as the confidence intervals did not reach 1 .

Table 3 Per-polyp sensitivity and false positives rate of experienced observers and novices

\begin{tabular}{|c|c|c|c|c|c|c|c|c|c|c|c|}
\hline \multirow{3}{*}{$\begin{array}{l}\text { Observer } \\
\text { Number }\end{array}$} & \multirow[b]{3}{*}{ Experience } & \multicolumn{6}{|c|}{ Per-polyp sensitivity (\%) } & \multicolumn{4}{|c|}{ Number of false positives } \\
\hline & & \multicolumn{3}{|l|}{$2 \mathrm{D}$} & \multicolumn{3}{|l|}{$3 \mathrm{D}$} & $2 \mathrm{D}$ & $3 \mathrm{D}$ & $2 \mathrm{D}$ & $3 \mathrm{D}$ \\
\hline & & $\geq 6 \mathrm{~mm}$ & $6-9 \mathrm{~mm}$ & $\geq 10 \mathrm{~mm}$ & $\geq 6 \mathrm{~mm}$ & $6-9 \mathrm{~mm}$ & $\geq 10 \mathrm{~mm}$ & \multicolumn{2}{|c|}{$\geq 6 \mathrm{~mm}$} & \multicolumn{2}{|c|}{$\geq 10 \mathrm{~mm}$} \\
\hline 2 & Experienced & $80(70-87)$ & $65(47-79)$ & $91(79-96)$ & $82(74-88)$ & $76(61-86)$ & $87(77-93)$ & 11 & 7 & 2 & 0 \\
\hline 6 & Experienced & $78(69-85)$ & $57(43-70)$ & $92(79-98)$ & $78(67-86)$ & $65(48-79)$ & $87(73-94)$ & 9 & 12 & 1 & 2 \\
\hline \multicolumn{2}{|c|}{ Mean experienced observers } & $79(70-86)$ & $61(46-74)$ & $92(79-97)$ & $80(72-86)$ & $70(57-81)$ & $87(75-93)$ & 10 & 10 & 2 & 1 \\
\hline 1 & Novice & $63(53-71)$ & $42(27-58)$ & $77(66-85)$ & $77(67-85)$ & $67(49-81)$ & $85(74-91)$ & 2 & 4 & 1 & 1 \\
\hline 3 & Novice & $59(50-67)$ & $38(24-54)$ & $74(59-84)$ & $63(54-72)$ & $46(31-62)$ & $75(61-86)$ & 7 & 8 & 1 & 3 \\
\hline 4 & Novice & $56(45-66)$ & $27(14-45)$ & $75(59-87)$ & $66(55-75)$ & $41(23-61)$ & $83(68-92)$ & 8 & 10 & 3 & 4 \\
\hline 5 & Novice & $53(44-62)$ & $24(12-42)$ & $74(61-83)$ & $68(58-76)$ & $46(29-64)$ & $83(74-90)$ & 2 & 9 & 1 & 3 \\
\hline 7 & Novice & $29(21-39)$ & $8(3-23)$ & $45(31-57)$ & $53(43-64)$ & $32(19-50)$ & $68(49-82)$ & 4 & 13 & 4 & 7 \\
\hline 8 & Novice & $49(35-64)$ & $22(9-43)$ & $70(54-82)$ & $64(52-75)$ & $49(32-65)$ & $76(61-86)$ & 14 & 18 & 7 & 2 \\
\hline \multicolumn{2}{|c|}{ Mean novice observers } & $51 *(44-59)$ & $27 *(17-39)$ & $69 *(59-77)$ & $65 *(57-73)$ & $46^{*}(33-60)$ & $78 *(68-86)$ & 6 & 10 & 3 & 3 \\
\hline
\end{tabular}

Individual per-polyp sensitivity is stratified for polyp size; $95 \%$ confidence intervals are between brackets

*Denotes statistically significant difference between the review methods 
Table 4 Per-patient sensitivity and specificity of experienced observers and novices

\begin{tabular}{|c|c|c|c|c|c|c|c|c|c|}
\hline \multirow{2}{*}{\multicolumn{2}{|c|}{ Observer }} & \multicolumn{4}{|c|}{ Per-patient sensitivity (\%) } & \multicolumn{4}{|l|}{ Specificity (\%) } \\
\hline & & \multicolumn{2}{|l|}{$2 \mathrm{D}$} & \multicolumn{2}{|l|}{$3 \mathrm{D}$} & \multicolumn{2}{|l|}{$2 \mathrm{D}$} & \multicolumn{2}{|l|}{$3 \mathrm{D}$} \\
\hline Number & Experience & $\geq 6 \mathrm{~mm}$ & $\geq 10 \mathrm{~mm}$ & $\geq 6 \mathrm{~mm}$ & $\geq 10 \mathrm{~mm}$ & $\geq 6 \mathrm{~mm}$ & $\geq 10 \mathrm{~mm}$ & $\geq 6 \mathrm{~mm}$ & $\geq 10 \mathrm{~mm}$ \\
\hline 2 & Experienced & $88(79-97)$ & $97(91-100)$ & $86(76-96)$ & $91(81-100)$ & $95(87-100)$ & $97(92-100)$ & $91(79-100)$ & $100(100-100)$ \\
\hline 6 & Experienced & $84(74-94)$ & $97(91-100)$ & $80(69-91)$ & $91(82-100)$ & $95(87-100)$ & $100(100-100)$ & $91(79-100)$ & $95(88-100)$ \\
\hline \multicolumn{2}{|c|}{$\begin{array}{c}\text { Mean values } \\
\text { experienced } \\
\text { observers }\end{array}$} & $86(79-93)$ & $97(93-100)$ & $83(76-90)$ & $91(84-98)$ & $95(89-100)$ & $99(96-100)$ & $91(82-99)$ & $97(94-100)$ \\
\hline 1 & Novice & $71(58-84)$ & $82(69-95)$ & $85(75-95)$ & $91(81-100)$ & $100(100-100)$ & $97(92-100)$ & $91(79-100)$ & $97(92-100)$ \\
\hline 3 & Novice & $74(62-86)$ & $85(73-97)$ & $72(60-84)$ & $79(66-93)$ & $91(79-100)$ & $100(100-100)$ & $95(87-100)$ & $95(88-100)$ \\
\hline 4 & Novice & $68(55-81)$ & $85(73-97)$ & $74(62-86)$ & $91(82-100)$ & $9587-100)$ & $95(88-100)$ & $100(100-100)$ & $95(88-100)$ \\
\hline 5 & Novice & $70(57-83)$ & $88(77-99)$ & 78 (67-89) & $91(82-100)$ & $100(100-100)$ & $97(92-100)$ & $91(79-100)$ & $97(92-100)$ \\
\hline 7 & Novice & $42(28-56)$ & $59(42-75)$ & $72(60-84)$ & 85 (73-97) & $100(100-100)$ & $97(92-100)$ & $91(79-100)$ & $95(88-100)$ \\
\hline 8 & Novice & $62(48-76)$ & $81(67-95)$ & $68(55-81)$ & 81 (67-95) & $95(87-100)$ & $95(88-100)$ & 77 (60-95) & $95(88-100)$ \\
\hline \multicolumn{2}{|c|}{$\begin{array}{l}\text { Mean values novice } \\
\text { observers }\end{array}$} & $64(59-70)$ & $80(74-86)$ & $75 *(70-80)$ & $86^{*}(82-91)$ & $97(94-100)$ & 97 (95-99) & $91(86-96)$ & $96(93-98)$ \\
\hline
\end{tabular}

Individual per-patient sensitivity and specificity are stratified for polyp size; 95\% confidence interval are between brackets) *Denotes statistically significant difference between the review methods

Floating debris (Fig. 4) and holes in the colon wall (Fig. 5) were important causes of artefacts in PEC3D (Table 6). According to the observers, floating debris in particular may hinder the diagnostic accuracy by covering polyps $6 \mathrm{~mm}$ or larger. In PU2D virtually no disturbing artefacts were reported.

\section{Discussion}

This study shows that novice observers (compared with experienced observers) have a higher sensitivity in limited prepared patients when using PEC3D compared with PU2D. The higher sensitivity comes without a statistically significant lower specificity. On average more review time was needed for PEC3D. Paradoxically, despite its superior performance in polyp detection, the image quality of PEC3D was rated significantly less than for PU2D.

Recently, two comparative studies of primary 2D and primary 3D review methods have addressed the merit of both techniques $[11,12]$. As in this study, more polyps of $6 \mathrm{~mm}$ or larger were detected using primary $3 \mathrm{D}$, although in only one study the difference was statistically significant [11]. The reason for this superior sensitivity may be that abnormalities are visible to the observer for longer. Secondly, polyps that may have a similar appearance to folds on 2D are easier to distinguish from folds in $3 \mathrm{D}$. However, the fact that primary $3 \mathrm{D}$ is often not used in CTC is probably based on practical grounds such as long review time and high computer requirements associated with this review technique in the past.
Compared with the abovementioned previous studies, in this study patients underwent a limited bowel preparation. There is a risk that the reported superiority of $3 \mathrm{D}$ in polyp detection would be neutralized by the reduction of visible colonic surface. In this study we report a large number of at least partially submerged polyps. This increases the risk of overlooking polyps $[21,22]$. This is the reason why electronic cleansing was used in the 3D examinations.

Electronic cleansing has been subject to study for several years now [16, 23-27]. Recently two comparative studies of electronic cleansing were published. In both studies electronic cleansing had an additional value in terms of sensitivity for some observers $[23,24]$. In this study we assumed it had an additional value as well: nearly half of the polyps were in at least one position at least partially covered by faecal material (Table 2).

Specific artefacts of electronic cleansing are described in the literature [13] e.g. ridges, pseudopolyps due to partial volume effect and floating debris due to untagged faecal material. These may be the reason that electronic cleansing for primary CTC evaluation has not often been used. The electronic cleansing algorithm we used in this study was specially designed to overcome artefacts of distracting 'ridges' emanating from locations where air, soft tissue and tagged material meet [16]. These ridges were in fact noted by none of the observers in this study.

Floating debris, though, was detected in the majority of patients examined in PEC3D (Table 6). An important cause of debris is noise due to heterogeneously or insufficiently tagged stool. The three patients were excluded because of these artefacts. These artefacts stress the fact that more than just a good cleansing algorithm is important in order to 
Table 5 Mean rating of diagnostic quality

\begin{tabular}{lll}
\hline $\begin{array}{l}\text { Image quality of the } \\
\text { examinations }\end{array}$ & $\begin{array}{l}\text { 2D } \\
\text { Percentage of } \\
\text { the examinations }\end{array}$ & $\begin{array}{l}\text { 3D } \\
\text { Percentage of } \\
\text { the examinations }\end{array}$ \\
\hline $\begin{array}{l}\text { Not diagnostic, polyps } 10 \mathrm{~mm} \\
\text { or larger can be missed }\end{array}$ & 1 & 4 \\
$\begin{array}{l}\text { Diagnostic with many artefacts, } \\
\text { polyps 6-9 mm can be missed }\end{array}$ & 18 \\
$\begin{array}{l}\text { Diagnostic with a small } \\
\text { number of artefacts, polyps }\end{array}$ & 20 & 53 \\
$\begin{array}{l}6 \text { mm or larger cannot be } \\
\text { missed }\end{array}$ & \\
$\begin{array}{l}\text { Diagnostic without artefacts } \\
\text { Unknown }\end{array}$ & 69 & 19 \\
\hline
\end{tabular}

Table displays the percentage of PU2D and PEC3D examinations in the various rating categories. The percentage represented the mean rating of all eight observers in 72 patients

achieve good 3D image quality i.e. a good tagging regimen, good patient compliance and good CT parameters. However, the three excluded patients were rated in PU2D by one to three observers "not diagnostic" as well. This stresses the fact that although $3 \mathrm{D}$ is more susceptible to tagging artefacts, 2D suffers from heterogeneous or insufficient tagging as well.

Artefacts were seen in the majority of patients reviewed in PEC3D. To be able to easily distinguish artefacts from polyps, it is important to be able to correlate electronically cleansed 3D images with complementary original uncleansed 2D images. This combination has limited the number of false positive findings when using PEC3D (Table 3). Using PEC3D did not statistically decrease specificity for any observer group at any size per-patient threshold i.e. true negative patients were not erroneously classified using PEC3D.

We have not studied a primary $3 \mathrm{D}$ reading method without electronic cleansing or primary $2 \mathrm{D}$ with electronic cleansing. Although interesting from a methodological point of view, we think that these approaches are not meaningful; the former is not since a large number of (partially) submerged polyps are prevented from being detected because they are otherwise covered by faecal material; the second approach is not since there is no need to electronically remove faecal material that can already be distinguished from colonic structures. However, one study [23] demonstrated an additional value in terms of polyp detection of cleansing in a 2D approach. An important difference compared with our study is the nature of the preparation: barium instead of iodine and no low-fibre diet. This results in more adherent and solid stool that is "mentally" more difficult to read than the quiet homogeneous fluid levels seen in our patient population. Therefore, electronic cleansing may prevent reader fatigue in this patient population.
The mean difference between PU2D and PEC3D was in accordance with the expected difference between both techniques. However, the expected baseline sensitivity for polyps $6 \mathrm{~mm}$ or larger was higher (70\%) than the actual measured sensitivity (51\%). This had consequences for the statistical power of the comparison; however, statistical significance was still reached for the group of inexperienced readers.

The higher per-polyp sensitivity of PEC3D mainly concerned polyps 6-9 $\mathrm{mm}$ (Table 3 ). The prevalence of adenomas with advanced features (i.e. villous components or high-grade dysplasia) in this size category tends to be low [28]. The joint guideline of the American Cancer Society, the US Multi-Society Task Force on Colorectal Cancer and the American College of Radiology recommends colonoscopy and polypectomy for polyps $6 \mathrm{~mm}$ or larger [1]. Thus, polyps in this size category may not be neglected.

The novice observers were trained according to the recommendations of the American College of Radiology and the European Society of Gastrointestinal and Abdominal Radiology [29, 30]; 50 CTC studies with 20-50\% prevalence with personal feedback on all false positive and negative findings for polyps $6 \mathrm{~mm}$ or larger [29]. The response to training, though, is unpredictable and competence cannot be assumed after 50 cases [31]. In this study the two experienced observers (350 CTCs or more) outperformed the six novice observers. Thus, it is likely that the optimum number of training cases is more than 50 , as suggested earlier [32]. However, the difference between these levels of experience in PEC3D is less than PU2D. So,

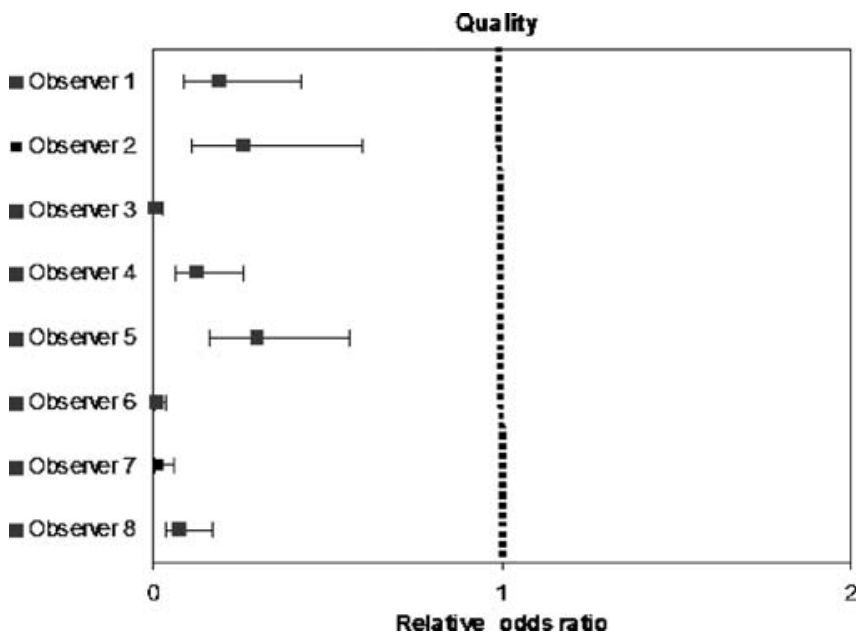

Fig. 3 Image quality assessment showing regression coefficients of primary electronically cleansed 3D (PEC3D). It estimates the change in the log transformed diagnostic odds ratio (DOR) compared with primary uncleansed 2D (PU2D). A lower relative diagnostic odds ratio (RDOR) implies inferior image quality. Confidence intervals not reaching 1 indicate significant inferiority. Thus, this figure shows that all observers rated the image quality of PEC3D significantly less qualitative than PU2D 


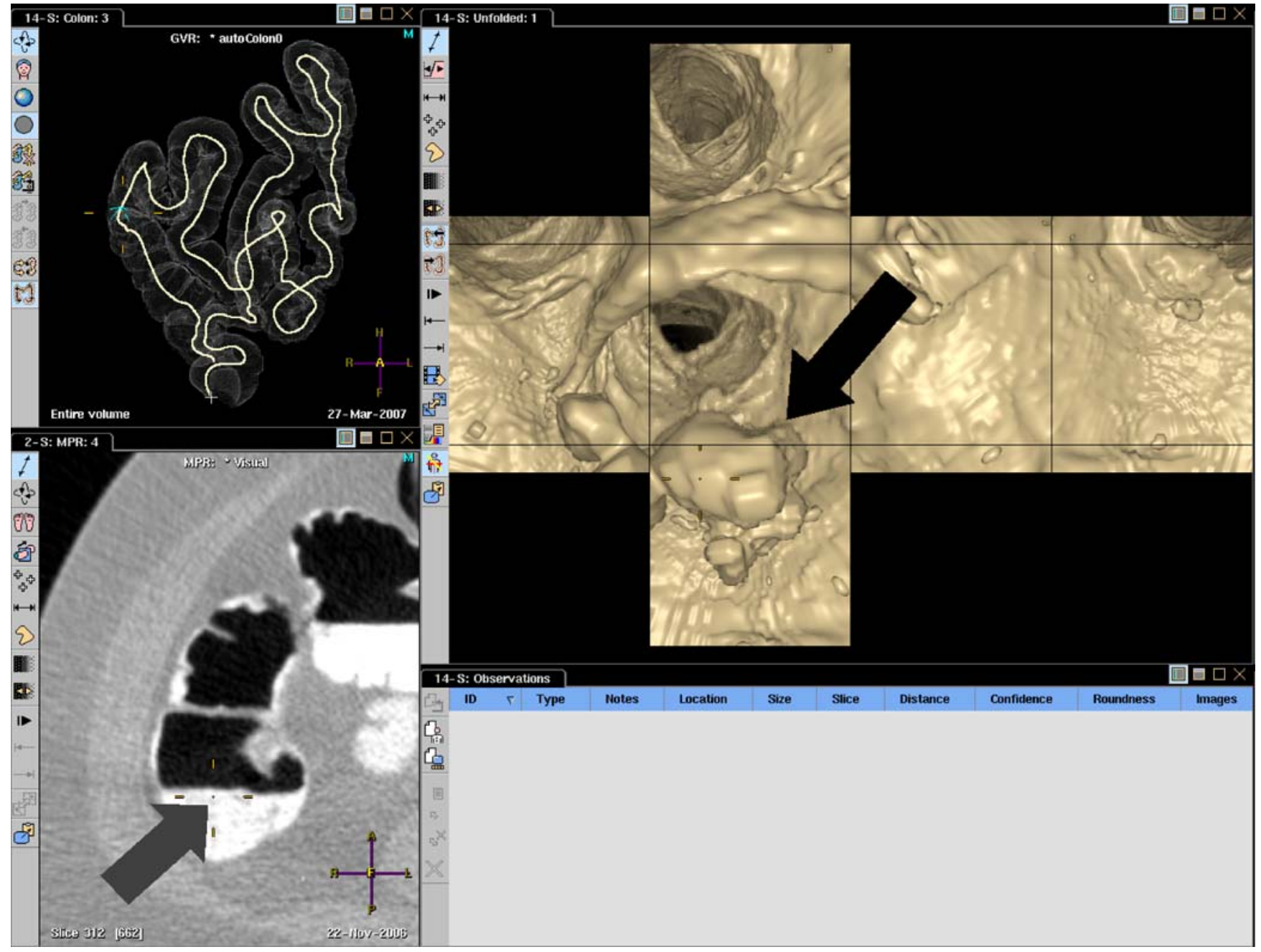

Fig. 4 Floating debris (black arrow) as a result of incomplete electronic cleansing of inhomogeneously tagged faecal material (grey arrow on $2 \mathrm{D}$ image)

in the phase of familiarization with CTC primary cleansed $3 \mathrm{D}$ is advantageous.

The group of experienced readers consisted of two observers compared with the group of inexperience readers that consisted of six observers. In general, experienced observers show less difference in polyp detection between review methods [33] and will perform better when compared with inexperienced readers. Therefore, we expected the differences between both review methods to be insignificant in a very experienced observer group. This was confirmed by the two experienced reviewers in this study who performed very well using both methods. Thus, we think that although interesting from a methodological point of view adding four extra reviewers would not change the conclusion of this study.

A computer-aided detection algorithm has not been used in this study. Its effect will be twofold when applied to electronically cleansed data: the number of detectable polyps will increase as well as the number of detected artefacts. Since these effects are not yet balanced, this will be subject to further research.

This study has limitations. First the prevalence of polyps in this FOBT positive population was fairly high compared with an asymptomatic screening population. This may limit the generalization to an average risk screening population.

Secondly, in this patient population we used a low-dose scan protocol combined with sub-millimetre slices. Noise in the images may have limited the quality of the cleansing since faecal material appeared less homogeneous. Still, the quality of the images was rated diagnostic in the vast majority of the cases i.e. only three cases were excluded.

Thirdly, all patients had been prepared with oral iodine tagging resulting in a fairly homogeneously tagged colon content. Probably any electronic cleansing algorithm will perform optimal with homogeneously tagged stool [27]. Accordingly, we expect the algorithm to be better suited for removing pools of (iodine) fluid compared with adherent heterogeneous faecal residue encountered in barium tagging. 


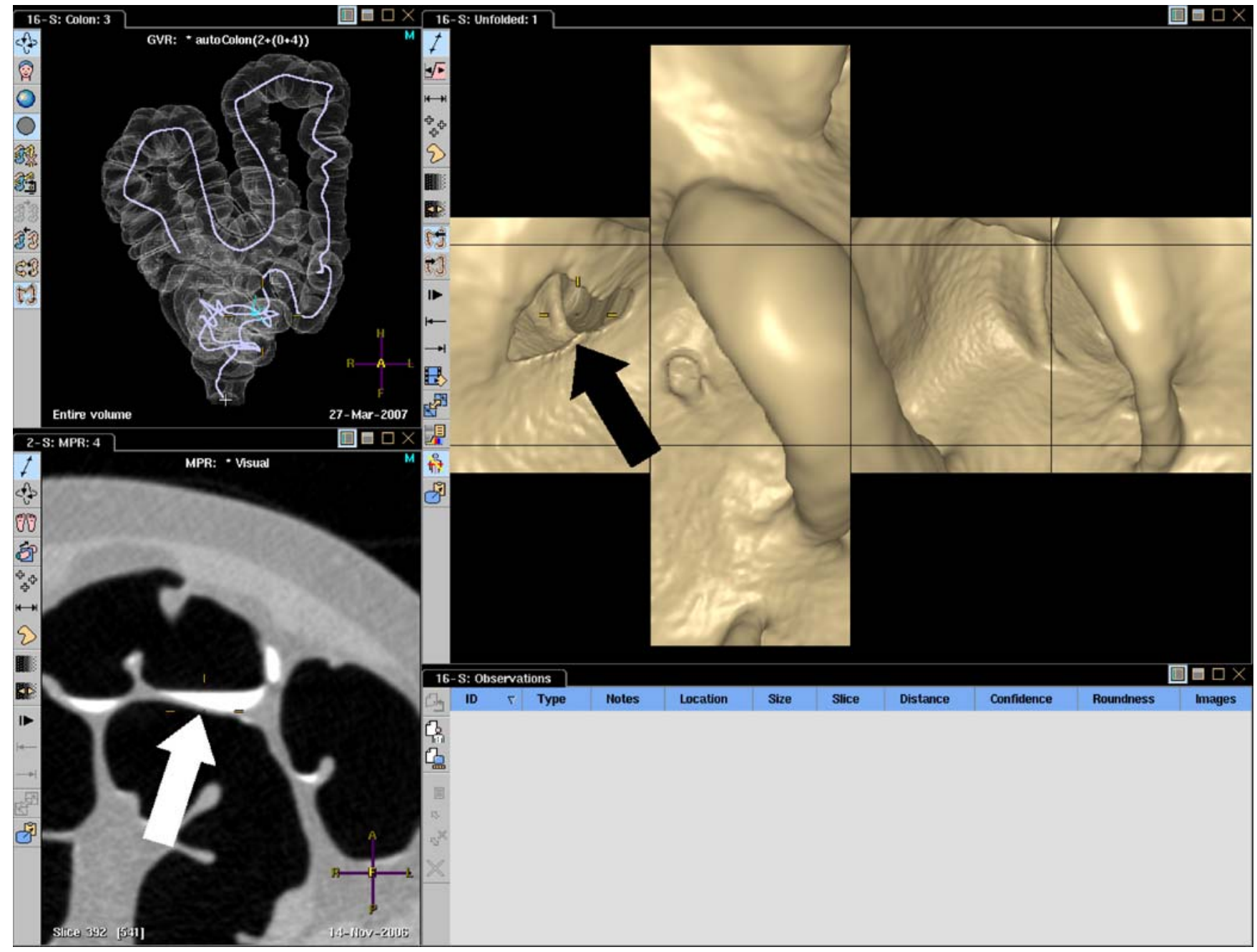

Fig. 5 Hole in the wall on the 3D image (black arrow). The white arrow indicates the corresponding colon wall in 2D. After electronic cleansing, the colon wall has become so thin that virtual holes appear between two air-containing structures

Table 6 Number of patients with artefacts

\begin{tabular}{llll}
\hline Artefacts & Number of patients & $\begin{array}{l}\text { Number of patients having artefacts } \\
\text { classified as "disturbing, can cover } \\
\text { polyps } 6 \text { mm or larger" }\end{array}$ & $\begin{array}{l}\text { Number of patients having artefacts } \\
\text { classified as "disturbing, can cover } \\
\text { polyps } 10 \text { mm or larger" }\end{array}$ \\
\hline $\begin{array}{l}\text { Primary electronically cleansed } \\
\text { Floating debris }\end{array}$ & $55(76 \%)$ & $18(25 \%)$ & $1(1 \%)$ \\
Holes in the colon wall & $39(54 \%)$ & $2(3 \%)$ & $0(0 \%)$ \\
Windmill artefacts & $1(1 \%)$ & $0(0 \%)$ & $0(0 \%)$ \\
Ridges & $0(0 \%)$ & $0(0 \%)$ & $0(0 \%)$ \\
Primary uncleansed 2D & & & $0(0 \%)$ \\
Windmill artefacts & $2(3 \%)$ & $0(0 \%)$ & $0(0 \%)$ \\
Insufficient faecal tagging & $2(3 \%)$ & $1(1 \%)$ & \\
\hline
\end{tabular}

Table displays the number of patients with artefacts observed by at least four observers, the number of patients having artefacts classified by at least four observers as "disturbing, can cover polyps $6 \mathrm{~mm}$ or larger" and the number of patients having artefacts classified by at least four observers as "disturbing, can cover polyps $10 \mathrm{~mm}$ or larger" 
Fourthly, before this study the experienced observers had evaluated all patients in the framework of a comparative study of colonoscopy and CTC (http://rsna2008.rsna.org/ event display.cfm?em id=6012336). These patients were evaluated at least 1 year before this study with a primary $2 \mathrm{D}$ review method. In the period in between both studies at least 100 other CTC examinations were read. So, it is not likely that this has influenced the performance characteristics of the experienced observers.

Fifthly, in this study we have used an enhanced 3D display i.e. the unfolded cube display. The advantage of this technique is that it covers nearly all colonic mucosa without image distortion in a single fly-through [15], compared with the conventional 'endoscopic' view that needs a bidirectional fly-through to cover nearly all colonic mucosa. Therefore, our approach is a more time efficient method than a conventional 3D technique [15]. This may limit the generalizability of difference in review time, however not in accuracy.
In summary, we conclude that novice observers have a significantly higher sensitivity for the detection of clinically relevant polyps when using primary electronically cleansed $3 \mathrm{D}$ compared with primary $2 \mathrm{D}$. For experienced observers, who performed better overall, there is no difference between both methods. Specificity is not affected when using primary electronically cleansed 3D. Therefore we recommend primary electronically cleansed 3D for novice observers in evaluating CTC in patients that have undergone limited bowel preparation.

Open Access This article is distributed under the terms of the Creative Commons Attribution Noncommercial License which permits any noncommercial use, distribution, and reproduction in any medium, provided the original author(s) and source are credited.

Acknowledgments A grant was received from Philips Healthcare.

\section{References}

1. Levin B, Lieberman DA, McFarland B et al (2008) Screening and surveillance for the early detection of colorectal cancer and adenomatous polyps, 2008: a joint guideline from the American Cancer Society, the US Multi-Society Task Force on Colorectal Cancer, and the American College of Radiology. Gastroenterology 134:1570-1595

2. van Gelder RE, Birnie E, Florie J et al (2004) CT colonography and colonoscopy: assessment of patient preference in a 5-week follow-up study. Radiology 233:328-337

3. Iannaccone R, Laghi A, Catalano $\mathrm{C}$ et al (2004) Computed tomographic colonography without cathartic preparation for the detection of colorectal polyps. Gastroenterology 127:13001311

4. Jensch S, de Vries AH, Peringa J et al (2008) CT colonography with limited bowel preparation: performance characteristics in an increased-risk population. Radiology 247:122-132

5. Lefere P, Gryspeerdt S, Marrannes J, Baekelandt M, van HB (2005) CT colonography after fecal tagging with a reduced cathartic cleansing and a reduced volume of barium. AJR Am J Roentgenol 184:1836-1842
6. Iafrate F, Hassan C, Zullo A et al (2008) CT colonography with reduced bowel preparation after incomplete colonoscopy in the elderly. Eur Radiol 18:1385-1395

7. Gryspeerdt S, Lefere P, Herman M et al (2005) CT colonography with fecal tagging after incomplete colonoscopy. Eur Radiol 15:1192-1202

8. Gluecker TM, Johnson CD, Harmsen WS et al (2003) Colorectal cancer screening with CT colonography, colonoscopy, and double-contrast barium enema examination: prospective assessment of patient perceptions and preferences. Radiology 227:378-384

9. Rex DK (2002) Current colorectal cancer screening strategies: overview and obstacles to implementation. Rev Gastroenterol Disord 2(Suppl 1):S2-S11

10. Weitzman ER, Zapka J, Estabrook B, Goins KV (2001) Risk and reluctance: understanding impediments to colorectal cancer screening. Prev Med 32:502513

11. Pickhardt PJ, Lee AD, Taylor AJ et al (2007) Primary 2D versus primary 3D polyp detection at screening CT colonography. AJR Am J Roentgenol 189:1451-1456

12. van Gelder RE, Florie J, Nio CY et al (2006) A comparison of primary twoand three-dimensional methods to review CT colonography. Eur Radiol 17:1181-1192
13. Pickhardt PJ, Choi JH (2003) Electronic cleansing and stool tagging in CT colonography: advantages and pitfalls with primary three-dimensional evaluation. AJR Am J Roentgenol 181:799-805

14. van Rossum L, van Rijn A, Laheij R et al (2008) Random comparison of Guaiac and immunochemical fecal occult blood tests for colorectal cancer in a screening population. Gastroenterology 135(1):82-90

15. Vos FM, van Gelder RE, Serlie IW et al (2003) Three-dimensional display modes for CT colonography: conventional 3D virtual colonoscopy versus unfolded cube projection. Radiology 228:878-885

16. Serlie IW, Vos FM, Truyen R, Post FH, van Vliet LJ (2007) Classifying CT image data into material fractions by a scale and rotation invariant edge model. IEEE Trans Image Process 16:2891-2904

17. Walter Reed Army Medical Center. Walter Reed Army Medical Center (2005) http://wramc.vcscreen.com/training/ index.html. Accessed 23 Nov 2005

18. Fennerty MB, Davidson J, Emerson SS, Sampliner RE, Hixson LJ, Garewal HS (1993) Are endoscopic measurements of colonic polyps reliable. Am J Gastroenterol 88:496-500 
19. Gopalswamy N, Shenoy VN, Choudhry U et al (1997) Is in vivo measurement of size of polyps during colonoscopy accurate. Gastrointest Endosc 46:497-502

20. Pepe MS (2003) The statistical evaluation of medical tests for classification and prediction. Oxford University Press, New York, pp 35-65

21. Doshi T, Rusinak D, Halvorsen RA, Rockey DC, Suzuki K, Dachman AH (2007) CT colonography: false-negative interpretations. Radiology 244:165-173

22. Gluecker TM, Fletcher JG, Welch TJ et al (2004) Characterization of lesions missed on interpretation of CT colonography using a 2D search method. AJR Am J Roentgenol 182:881-889

23. Johnson CD, Manduca A, Fletcher JG et al (2008) Noncathartic CT colonography with stool tagging: performance with and without electronic stool subtraction. AJR Am J Roentgenol 190:361-366
24. Juchems MS, Ernst A, Johnson P, Virmani S, Brambs HJ, Aschoff AJ (2008) Electronic colon-cleansing for CT colonography: diagnostic performance. Abdom Imaging. doi: 10.1007/ s00261-008-9386-6

25. Serlie IW, de Vries AH, Vos FM et al (2008) Lesion conspicuity and efficiency of CT colonography with electronic cleansing based on a threematerial transition model. AJR Am J Roentgenol 191:1493-502

26. Wang Z, Liang Z, Li X et al (2006) An improved electronic colon cleansing method for detection of colonic polyps by virtual colonoscopy. IEEE Trans Biomed Eng 53:1635-1646

27. Zalis ME, Perumpillichira JJ, Magee C, Kohlberg G, Hahn PF (2006) Taggingbased, electronically cleansed CT colonography: evaluation of patient comfort and image readability. Radiology 239:149-159

28. Kim DH, Pickhardt PJ, Taylor AJ (2007) Characteristics of advanced adenomas detected at CT colonographic screening: implications for appropriate polyp size thresholds for polypectomy versus surveillance. AJR Am J Roentgenol 188:940-944
29. ACR practice guideline for the performance of computed tomography (CT) colonography in adults (2006) http:// www.acr.org/SecondaryMainMenu Categories/quality_safety/guidelines/ $\mathrm{dx} /$ gastro/ct colonography.aspx. Accessed 8 July 2008

30. Taylor SA, Laghi A, Lefere P, Halligan S, Stoker J (2007) European Society of Gastrointestinal and Abdominal Radiology (ESGAR): consensus statement on CT colonography. Eur Radiol 17:575-579

31. Taylor SA, Halligan S, Burling D et al (2004) CT colonography: effect of experience and training on reader performance. Eur Radiol 14:1025-1033

32. Spinzi G, Belloni G, Martegani A, Sangiovanni A, Del FC, Minoli G (2001) Computed tomographic colonography and conventional colonoscopy for colon diseases: a prospective, blinded study. Am J Gastroenterol 96:394-400

33. Johnson CD, Chen MH, Toledano AY et al (2008) Accuracy of CT colonography for detection of large adenomas and cancers. N Engl J Med 359:1207-1217 